
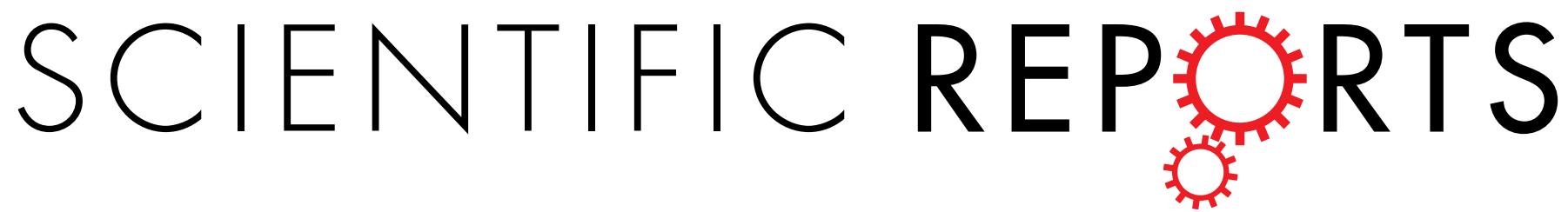

\title{
OPEN Modulation of electronic properties from stacking orders and spin-orbit coupling for 3R-type $\mathrm{MoS}_{2}$
}

Received: 14 January 2016

Accepted: 21 March 2016

Published: 07 April 2016
Xiaofeng Fan ${ }^{1,2}$, W. T. Zheng ${ }^{1}$, Jer-Lai Kuo ${ }^{3}$, David J. Singh ${ }^{4}$, C.O. Sun ${ }^{2} \&$ W. Zhu ${ }^{2}$

Two-dimensional crystals stacked by van der Waals coupling, such as twisted graphene and coupled graphene-BN layers with unusual phenomena have been a focus of research recently. As a typical representative, with the modulation of structural symmetry, stacking orders and spin-orbit coupling, transitional metal dichalcogenides have shown a lot of fascinating properties. Here we reveal the effect of stacking orders with spin-orbit coupling on the electronic properties of few-layer 3R-type $\mathrm{MoS}_{2}$ by first principles methods. We analyze the splitting of states at the top of valence band and the bottom of conduction band, following the change of stacking order. We find that regardless of stacking orders and layers' number, the spin-up and spin-down channels are evidently separated and can be as a basis for the valley dependent spin polarization. With a model Hamiltonian about the layer's coupling, the band splitting can be effectively analyzed by the coupling parameters. It is found that the stacking sequences, such as abc and abca, have the stronger nearest-neighbor coupling which imply the popular of periodic abc stacking sequence in natural growth of $\mathrm{MoS}_{2}$.

Few-layer sheets of hexagonal transition metal dichalcogenides (h-TMDs) based on two dimensional (2D) atomic layers with the chemical formula $\left(\mathrm{MX}_{2}\right)$ are with rich physical properties and has attracted broad attention for potential applications in electronic and optoelectronic devices ${ }^{1-4}$. The single-layer of $\mathrm{h}$-TMD is formed by the quasi-2D sheet of chemical bonded X-M-X, and with space group P- $6 \mathrm{~m} 2$. The formation of trigonal structure results in the absence of inversion symmetry which makes the demonstration of spin-orbit coupling in h-TMDs possible. Actually, the strong spin-orbit coupling due to the $\mathrm{d}$ orbitals of heavy metals offers the new opportunities to use these h-TMDs to study the coupled spin-valley 2D physics, such as spin- and valley- Hall effects and superconductivity ${ }^{5-13}$. In addition, the weak screening due to the low dimensional limitation has lead to tightly-bound excitons and enhanced electron-electron interactions ${ }^{14-16}$. These with the direct band gap in single-layer h-TMDs, also give rise to a lot of fascinating optical phenomena ${ }^{17-20}$, such as surface sensitive luminescence ${ }^{21,22}$ and strain-control of optical band gap ${ }^{23-27}$. Besides the symmetry and dimensionalit ${ }^{28}$, stacking orders also play an important role in the properties of few-layer layered materials ${ }^{29}$. The purpose of this paper is to present a detailed study of the role of stacking ways of layers and its effect on the electronic properties with the number of layers in the prototypical material, $\mathrm{MoS}_{2}$.

$\mathrm{MoS}_{2}$ is a layered compound and the layers are coupled by van der Waals $(\mathrm{vdW})$ interaction ${ }^{30}$. An overlooked feature of layered compound is there are a lot of polymorphs due to the different ways of stacking between layers. For example, the low-density phase $\mathrm{BN}$ with $\mathrm{sp}^{2}$-bonding is with layered structure and there are two popular polytypes, hexagonal phase $\mathrm{h}-\mathrm{BN}$ and layered rhombohedral phase $\mathrm{r}-\mathrm{BN}$, besides other stacking ways ${ }^{31}$. $\mathrm{MoS}_{2}$ is also with rich polytypism and the common phase of bulk $\mathrm{MoS}_{2}$ is $2 \mathrm{H}$-polytype ${ }^{32,33}$. Its unit cell is composed of a double-layer with space group P63/mmc. In this way, the inversion symmetry which is broken in single-layer $\mathrm{MoS}_{2}$ is restored in the bulk $2 \mathrm{H}-\mathrm{MoS}_{2}$. In addition to stable $2 \mathrm{H}$ phase, another stable phase, so called $3 \mathrm{R}$ phase with space group $\mathrm{R} 3 \mathrm{~m}$ is also popular and can be synthesized by controlled ways ${ }^{32,34,35}$. Besides three-dimensional spirals of atomic layered $\mathrm{MoS}_{2}$ by the way of growth, such as the CVD synthesis ${ }^{35}$, another popular way is the mechanical folding/stacking of single-layer $\mathrm{MoS}_{2}{ }^{28,36}$. The unit cell of $3 \mathrm{R}$ phase is composed of a triple-layer stacked. In this way, the inversion symmetry is broken in the bulk form which is different from $2 \mathrm{H}$ phase.

${ }^{1}$ College of Materials Science and Engineering, Jilin University, Changchun 130012, China. ${ }^{2}$ NOVITAS, School of Electrical and Electronic Engineering, Nanyang Technological University, 50 Nanyang Avenue, 639798, Singapore. ${ }^{3}$ Institute of Atomic and Molecular Sciences, Academia Sinica, Taipei, 10617, Taiwan. ${ }^{4}$ Department of Physics and Astronomy, University of Missouri, Columbia, Missouri 65211-7010, USA. Correspondence and requests for materials should be addressed toX.F.F. (email: xffan@jlu.edu.cn) orW.Z. (email: ewzhu@ntu.edu.sg) 
(a)

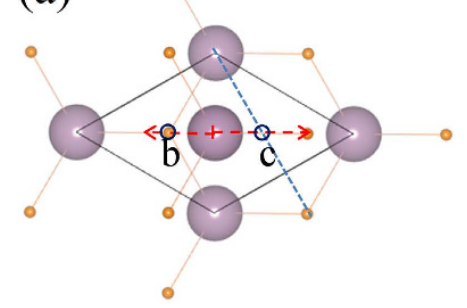

(b)

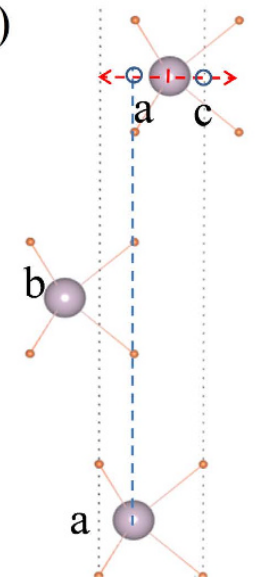

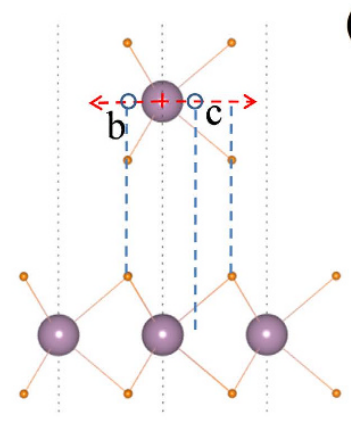

(c)

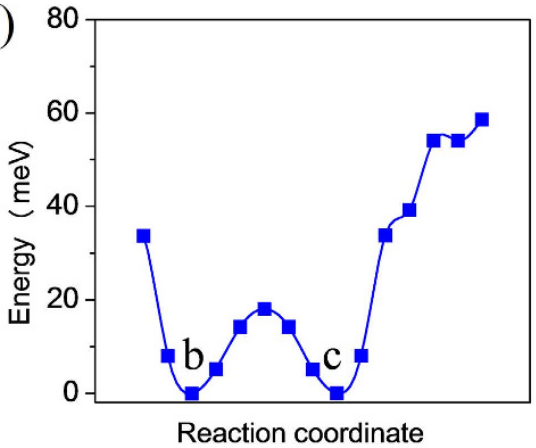

(d)

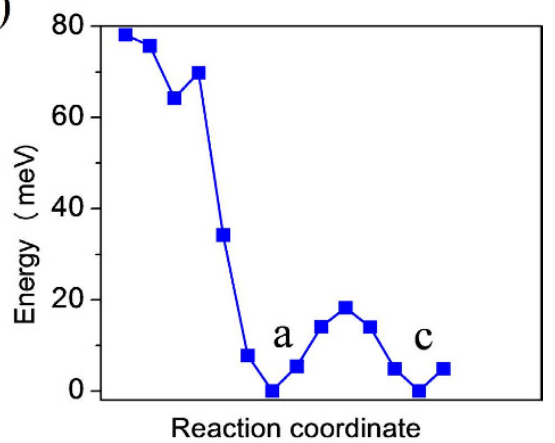

Figure 1. Schematic representation of (a) a unit cell of 2-layer $\mathrm{MoS}_{2}$ and (b) that of 3-layer $\mathrm{MoS}_{2}$ with abstacking for the lower two-layers and (c) the variation of total energy of 2-layer $\mathrm{MoS}_{2}$ due to the relative gliding of upper layer along the path indicated by the arrow in Fig. 1a and (d) that of 3-layer $\mathrm{MoS}_{2}$ due to the relative gliding of upper layer along the path indicated by the arrow in Fig. 1c.

Therefore, it is expected that 3R-type multi-layer $\mathrm{MoS}_{2}$ have different properties from $2 \mathrm{H}$-type $\mathrm{MoS}_{2}$, especially which related to the spin polarization ${ }^{37}$.

Here, we explore the effect of stacking orders with spin-orbit coupling (SOC) in combination with layer's coupling (LC) on few-layer 3R-type $\mathrm{MoS}_{2}$ using first principles methods. I explored the possible stacking ways for $\mathrm{MoS}_{2}$, firstly. Then the effect of different stacking orders on the electronic properties was considered for two-, three- and four-layer $\mathrm{MoS}_{2}$. We analyzed the splitting of states at the top of valence band at $\Gamma$ and $\mathrm{K}$ point (VB- $\Gamma$ and VB-K) and the bottom of conduction band along $\Lambda$ (CB- $\Lambda$ ) and at $K$ point $(\mathrm{CB}-\mathrm{K})$. The changes in splitting following the increase of the number of layers and change of stacking way were explored. It is found that the states of spin-up and spin-down near VB-K are separated obviously for few-layer 3R-type $\mathrm{MoS}_{2}$ whatever its number of layers is odd or even, and this is different from few-layer $2 \mathrm{H}$-type $\mathrm{MoS}_{2}$ in which valley polarization is complicated and is difficult to detect. We also found that LC has an important role to modulate the states near band gap, besides the contribution of SOC. The modulation of band splitting by LC was found to be changed, following the change of stacking orders.

\section{Results and Discussion}

Different stacking of $\mathrm{MoS}_{2}$ layers. For the layer-stacking of $\mathrm{MoS}_{2}$, there are two basic types of stacking ways. One type of stacking is the second layer of $\mathrm{MoS}_{2}$ is rotated $60^{\circ}$ along $\mathrm{z}$ axis relative to the first layer. Then the second layer glides on the surface of the first layer of $\mathrm{MoS}_{2}$ to find the energy minima. By this way, the most stable structure is where $S$ and Mo atoms of second layer are positioned above the Mo and $S$ atoms of first layer, respectively. It means there is inversion symmetry between the double layers. Such the periodic stacking of double layers results in $2 \mathrm{H}$-type $\mathrm{MoS}_{2}$.

Another type of stacking is there is no rotation between two layers. The second layer just glides on the surface of first layer, as shown in Fig. 1a. On the surface, there are two energy minima. For the two configurations, the Mo atoms of second layer are settled at b and c site, respectively. The both energy minima actually are equivalent, and there is an energy barrier of about $18 \mathrm{meV} / \mathrm{cell}$ between two energy minima in Fig. 1c. Obviously, if the Mo atom of second layer occupies the $b$ site and that of third layer occupies the $c$ site, such the periodic stacking of triple layers will form 3R-MoS . However, if the Mo atoms of first layer and second layer occupy the a and $b$ sites, the third layer will also be possible to occupy the a site or $\mathrm{c}$ site in Fig. 1c. This will result in different configurations whose symmetries are not equivalent. The gliding of third layer relative to second layer is simulated in Fig. $1 \mathrm{~d}$ and there are two minima on the energy surface which correspond to that the Mo atom of third layer is a site and c site (Fig. 1b), respectively. It is found that the energies of two minima haven't obvious different and the energy barrier between two minima is about $18 \mathrm{meV} /$ cell. Why the stacking orders have a tendency of abc-stacking sequence in the processes of natural growth has become a secret, like that on low-density phase BN. With the mechanical stacking or/and folding, both sequences aba and abc will be possible to appear, as observed in experiments. In 


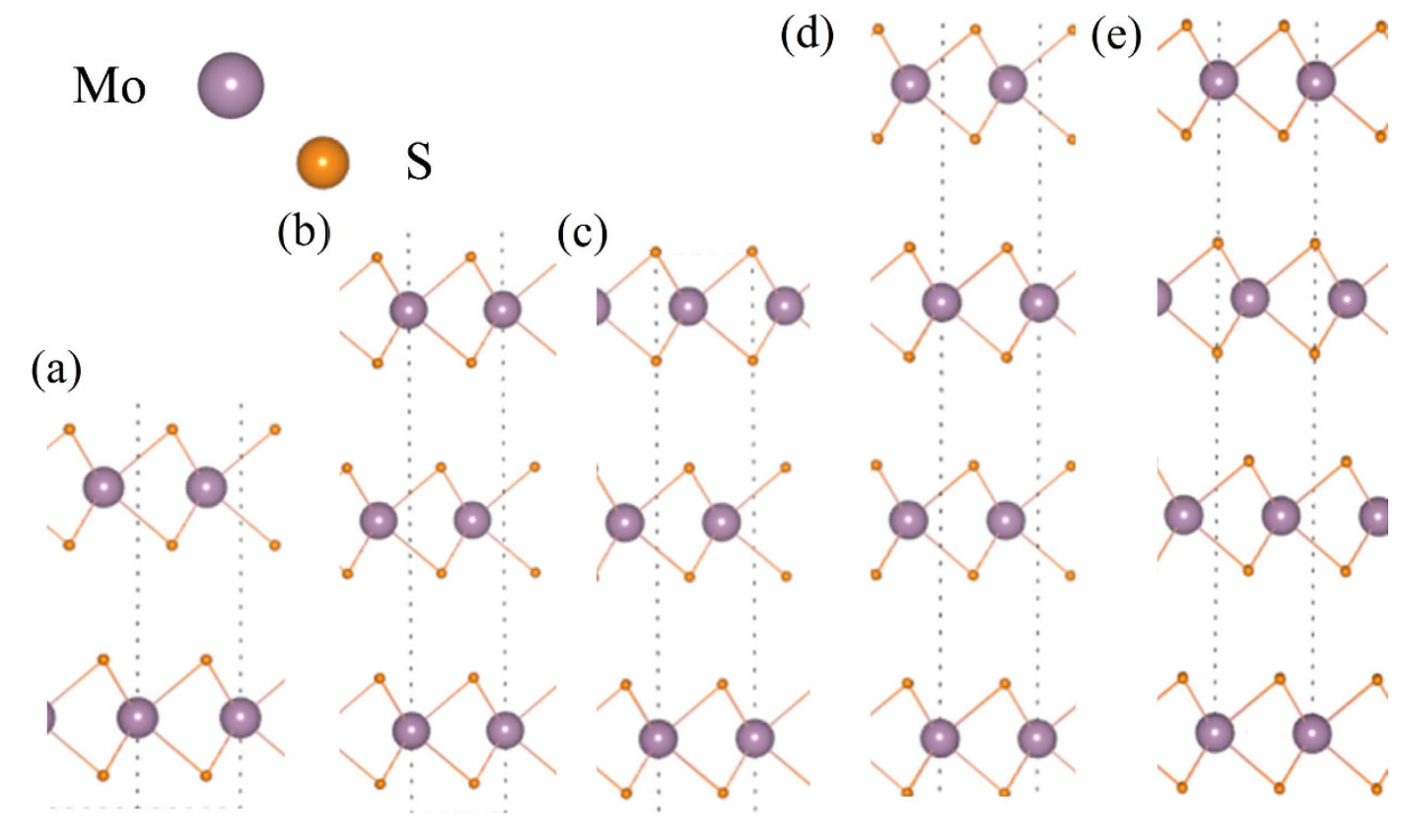

Figure 2. Crystal structures of multi-layer 3R-type $\mathrm{MoS}_{2}$ with different stacking ways including (a) 2-layer $\mathrm{MoS}_{2}$ with ab-stacking, (b) 3-layer $\mathrm{Mos}_{2}$ with aba-stacking, (c) 3-layer $\mathrm{MoS}_{2}$ with abc-stacking, (d) 4-layer $\mathrm{MoS}_{2}$ with abab-stacking and (e) 4-layer $\mathrm{MoS}_{2}$ with abca-stacking.

Fig. 2, multi-layer $\mathrm{MoS}_{2}$ with different stacking orders are shown to simulate the effect of stacking orders on electronic properties.

Electronic properties of double layer 3R-type $\mathrm{MoS}_{2}$. Layer's coupling makes the electronic properties of few-layer and bulk $\mathrm{MoS}_{2}$ different from single-layer $\mathrm{MoS}_{2}$. An obvious effect is the transition from direct band gap to indirect band gap, following the increase of layer's number ${ }^{38}$. As reported before, the main reason is the band splitting due to the LC results in the lift of valance band top at $\Gamma$ point ${ }^{39}$. It is well known that the states near band gap are mainly from Mo_d orbitals. Recently, it is noticed that there is obvious electronic occupation on $S$ atoms for the states near VB- $\Gamma$ and $\mathrm{CB}-\Lambda$. This results in the very sensitivity of the states near $\mathrm{VB}-\Gamma$ and $\mathrm{CB}-\Lambda$ to the layer's coupling and the ways of stacking. In Fig. 3a, there are obvious band splitting at $V B-\Gamma$ and $C B-\Lambda$, while the band splittings at VB-K and CB-K are small, without considering the SOC. From Table 1, they are $54 \mathrm{meV}$ and $61 \mathrm{meV}$, respectively.

The SOC from d orbitals of Mo has an important effect to the band structure and it is related to the symmetry of structure. The states at VB-K and CB- $\Lambda$ with the characteristic of $\mathrm{d}_{\mathrm{x}-\mathrm{y}}^{2}{ }^{2}$ and $\mathrm{d}_{\mathrm{xy}}$ orbitals of Mo have obvious band splitting with the SOC ${ }^{24}$. The states of VB- $\Gamma$ and CB-K are composed mostly by $\mathrm{d}_{\mathrm{z}}{ }^{2}$ orbitals of Mo. the sizable splitting at VB- $\Gamma$ and CB-K due to the SOC is not found. This may be because the $\mathrm{d}_{\mathrm{z}}^{2}$ orbital is related to the inversion symmetry which can result in the absence of SOC effect. In Fig. 3b, the splittings at CB- $\Lambda$ due to the SOC are $90 \mathrm{meV}$ and $61 \mathrm{meV}$ for the upper and down band and less than the splitting $(306 \mathrm{meV})$ between the main upper band and down band due to the LC. For the splitting near VB-K, the combination of LC and SOC makes it complicated in Fig. $3 \mathrm{c}$ and a model about the bands at VB-K is proposed in Fig. $3 \mathrm{~d}$.

In this model, the two upper bands are with spin-up and the splitting of both bands is due to the LC. The value of splitting $(61 \mathrm{meV})$ is same to that calculated without considering the SOC. The two down bands are with spin-down. The splitting between spin-up and spin-down bands is due to the SOC. For each band after the LC splitting, the splitting value due to the SOC is $149 \mathrm{meV}$ and similar to the SOC splitting of single-layer $\mathrm{MoS}_{2}$. As shown in Fig. 4, the characteristic of charge distribution of spin-up bands $(\sim|1 \uparrow\rangle$ and $\sim|2 \uparrow\rangle$ is obviously different from that of spin-down bands $(\sim|1 \downarrow\rangle$ and $\sim|2 \downarrow\rangle)$. For each spin channel, the charge densities of upper band and down band are mainly distributed in the upper layer and down layer, respectively. This also means the LC is weak for the states near VB-K.

Electronic structures of multi-layer 3R-type $\mathrm{MoS}_{2}$ with different stacking ways. For three-layer $\mathrm{MoS}_{2}$, the stacking order sequences aba and abc are considered and the band structures are calculated in Fig. 5 . The band structures of both stacking configurations are almost similar with each other. The states at VB- $\Gamma, \mathrm{CB}-\Lambda$, CB-K and VB-K near band gap have been split into three bands. With the SOC, the spin-up and spin-down channels at VB-K are separated clearly in Fig. 5b,d. One obvious difference is the two upper bands are almost doubly degenerate with one down band for each spin channel of aba stacking order. For abc stacking order, the three bands of each spin channel are separated distinctly.

In order to see the effect of symmetry on the stacking order further, the stacking orders of four-layer $\mathrm{MoS}_{2}$ are analyzed by considering the order sequences abab and abca. As shown in Fig. 6, the states at VB- $\Gamma$, CB- $\Lambda$, CB-K 
(a)

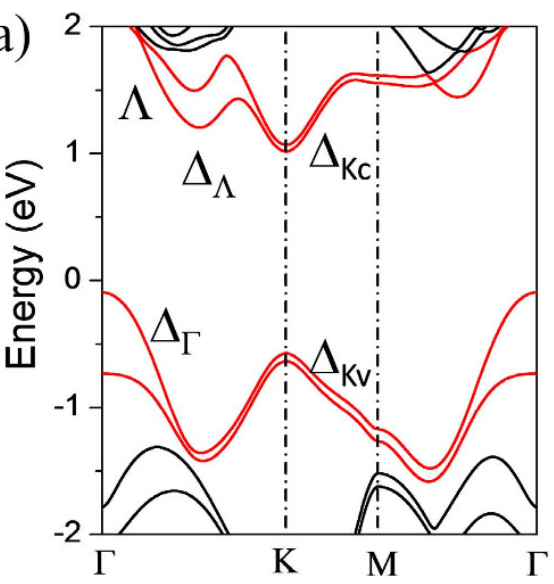

(c)

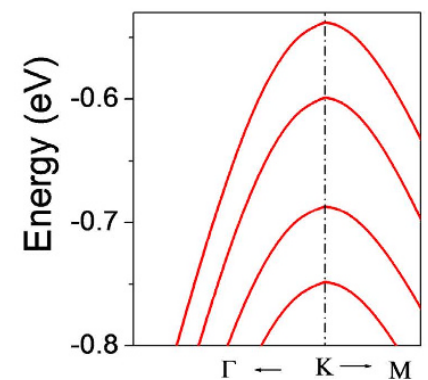

(b)

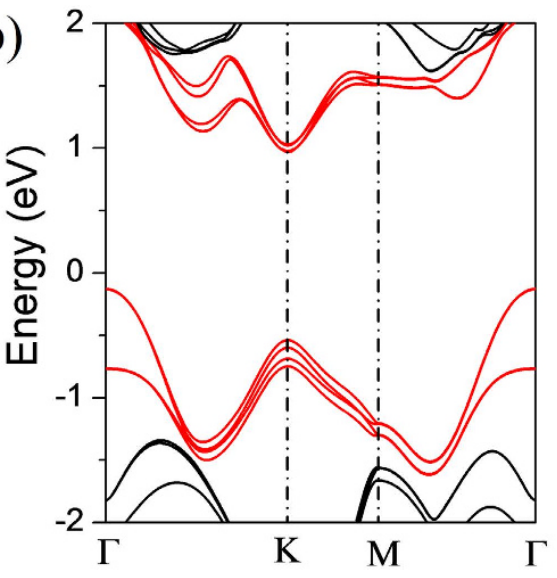

(d)

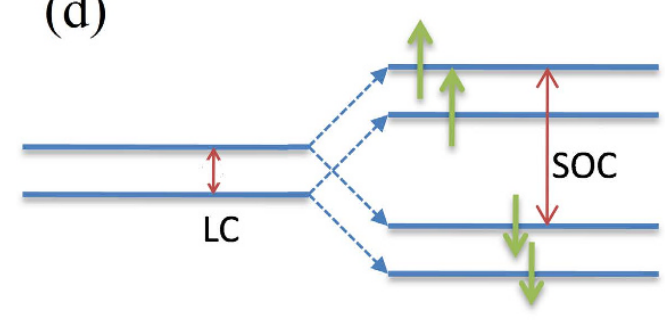

Figure 3. Band structures of 2-layer $\mathrm{MoS}_{2}$ with ab-stacking by calculated (a) without spin-orbit coupling (SOC) and (b) with SOC, (c) band structure from (b) plotted with two directions $\mathrm{K} \rightarrow \Gamma$ and $\mathrm{K} \rightarrow \mathrm{M}$ for the top of valance band near $k$ point, and (d) schematic of valance band splitting of the top of valance band at $K$ point due to SOC and layer's coupling (LC) in band structure of 2-layer $\mathrm{MoS}_{2}$.

\begin{tabular}{|c|c|c|c|c|}
\hline Stacking way & VB- $\Gamma$ & $\mathrm{CB}-\Lambda$ & CB-K & VB-K \\
\hline \multicolumn{5}{|l|}{$2 \mathrm{~L}-\mathrm{ab}$} \\
\hline$\Delta$ & 0.6389 & 0.3058 & 0.0543 & 0.0611 \\
\hline \multicolumn{5}{|l|}{$3 \mathrm{~L}$-aba } \\
\hline$\Delta_{1}$ & 0.2732 & 0.2699 & 0.0554 & 0.0079 \\
\hline$\Delta_{2}$ & 0.5324 & 0.1377 & 0.0011 & 0.0604 \\
\hline$\Delta$ & 0.8057 & 0.4076 & 0.0565 & 0.0683 \\
\hline \multicolumn{5}{|l|}{$3 \mathrm{~L}-\mathrm{abc}$} \\
\hline$\Delta_{1}$ & 0.3052 & 0.2278 & 0.0369 & 0.0549 \\
\hline$\Delta_{2}$ & 0.5228 & 0.1926 & 0.0535 & 0.0430 \\
\hline$\Delta$ & 0.8281 & 0.4204 & 0.0904 & 0.0979 \\
\hline \multicolumn{5}{|l|}{$4 \mathrm{~L}-\mathrm{abab}$} \\
\hline$\Delta_{1}$ & 0.1707 & 0.1572 & 0.0098 & 0.0081 \\
\hline$\Delta_{2}$ & 0.3067 & 0.2001 & 0.0426 & 0.0518 \\
\hline$\Delta_{3}$ & 0.4214 & 0.1017 & 0.0074 & 0.0115 \\
\hline$\Delta$ & 0.8988 & 0.4591 & 0.0597 & 0.0715 \\
\hline \multicolumn{5}{|l|}{$4 \mathrm{~L}-\mathrm{abca}$} \\
\hline$\Delta_{1}$ & 0.1810 & 0.1700 & 0.0409 & 0.0562 \\
\hline$\Delta_{2}$ & 0.3037 & 0.1798 & 0.0446 & 0.0449 \\
\hline$\Delta_{3}$ & 0.4317 & 0.1357 & 0.0549 & 0.0465 \\
\hline$\Delta$ & 0.9164 & 0.4855 & 0.1404 & 0.1476 \\
\hline
\end{tabular}

Table 1. Calculated band splitting values of the top of valance bands at $\Gamma$ and $K$ points (VB- $\Gamma$ and VB-K) and the bottom of conduction bands at $\Lambda$ and $K$ points (CB- $\Lambda$ and $C B-K$ ) for multi-layer 3R-type $\mathrm{MoS}_{2}$ including $2 \mathrm{~L}$-ab, $3 \mathrm{~L}$-aba, $3 \mathrm{~L}$-abc, $4 \mathrm{~L}$-abab and $4 \mathrm{~L}$-abca with the different layers and stacking orders. Note that $\Delta_{1}, \Delta_{2}$ and $\Delta_{3}$ represent the splitting values between the nearest-neighbor energy levels and $\Delta$ is for total splitting value. 
(a)
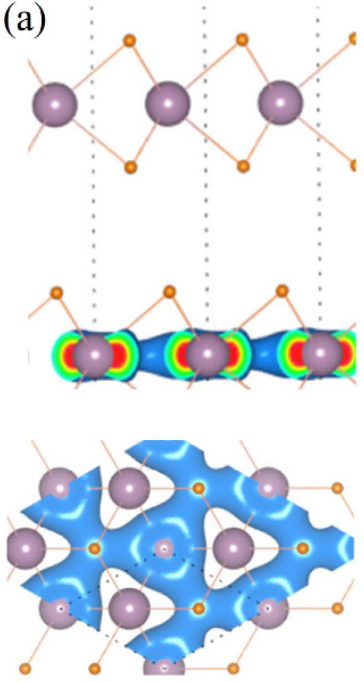

(b)
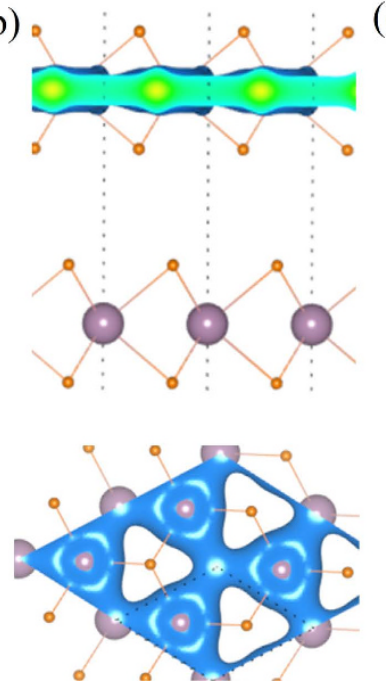

(c)
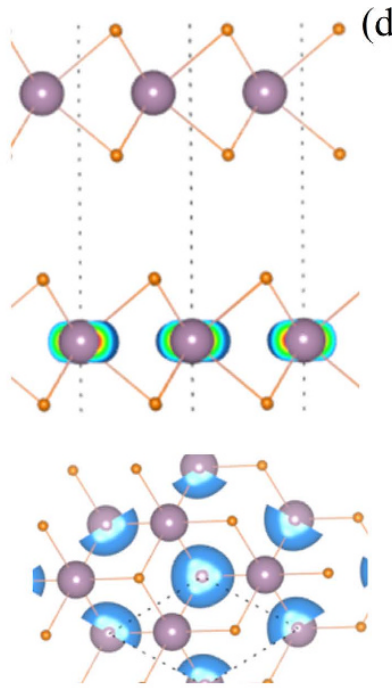

(d)
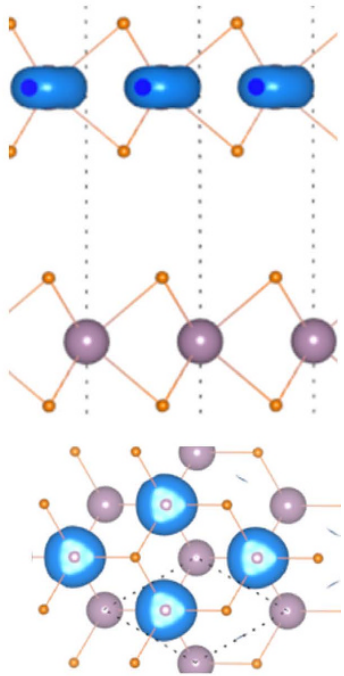

Figure 4. Isosurface of band-decomposed charge density of four states at the top of valance band of $\mathrm{K}$ point (in Fig. 3d) including the states (a) $\sim 1 \uparrow\rangle,(\mathbf{b}) \sim|2 \uparrow\rangle,(\mathbf{c}) \sim|1 \downarrow\rangle$, and (d) $\sim|2 \downarrow\rangle$ shown in Fig. 3d.
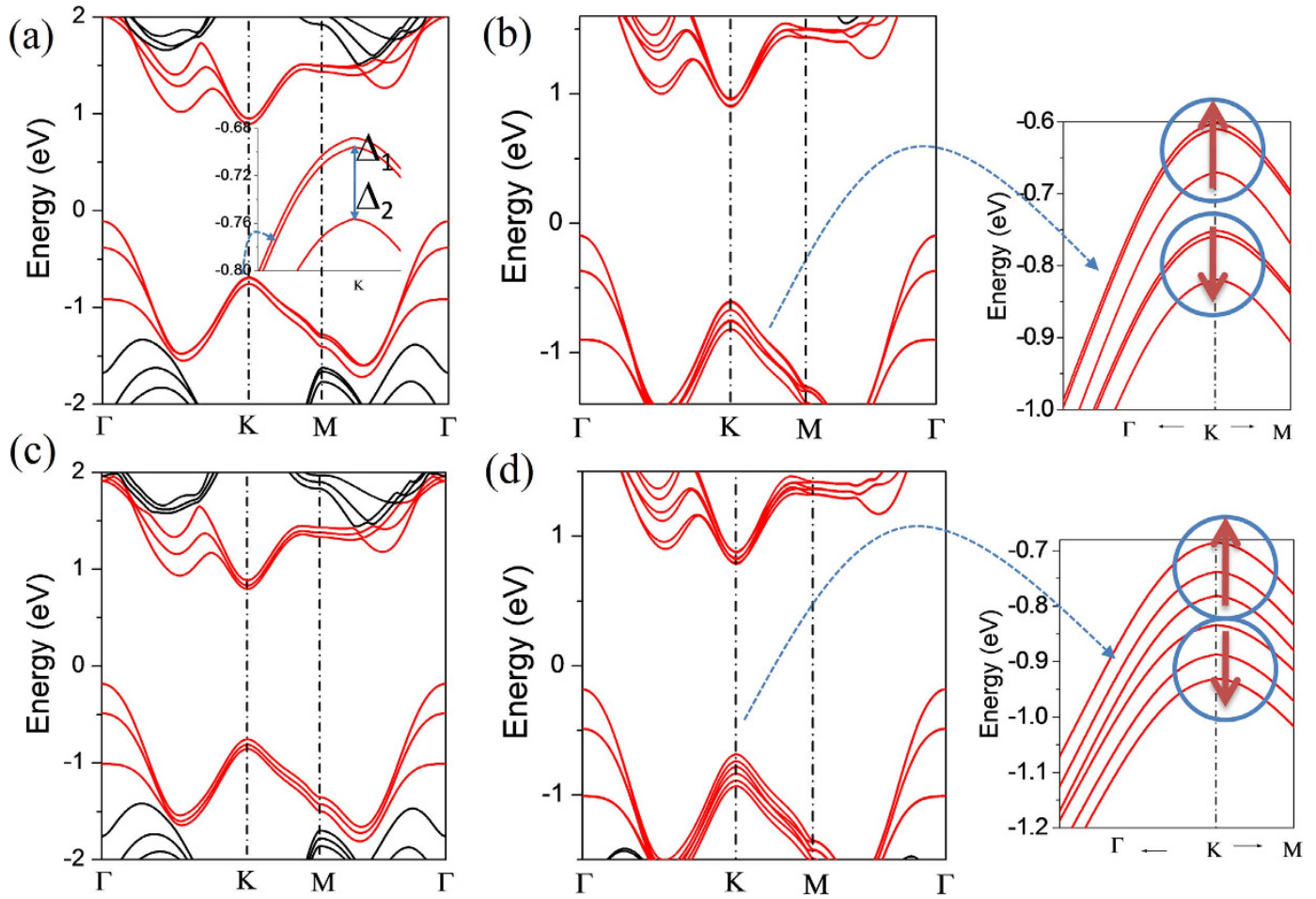

Figure 5. Band structures of 3-layer $\mathrm{MoS}_{2}$ with aba-stacking by calculated (a) without SOC and (b) with SOC, and with abc-stacking by calculated (c) without SOC and (d) with SOC. Note that in the inset of Fig. 5b and d, the band structure is plotted with two directions $\mathrm{K} \rightarrow \Gamma$ and $\mathrm{K} \rightarrow \mathrm{M}$ from the top of valance band near $\mathrm{K}$ point and the lengths for $\mathrm{K} \rightarrow \Gamma$ and $\mathrm{k} \rightarrow \mathrm{M}$ are the $1 / 10$ of total lengths in the two directions, respectively.

and VB-K near band gap have been split into four bands without considering the SOC. As the case of two-layer shown in Fig. $\mathrm{S} 1$ of supplementary information, the splitting of main bands of states at VB- $\Gamma, \mathrm{CB}-\Lambda$ and $\mathrm{CB}-\mathrm{K}$ is controlled mainly by the LC effect. For each main band of states at CB- $\Lambda$ and CB-K, the splitting from the SOC effect is found. For different stacking orders and different layer numbers, this splitting phenomenon is similar. For both order sequences, the spin-up and spin-down channels are also separated clearly in Fig. $6 \mathrm{~b}$ and d. For each spin channel of abab stacking order at the states of VB-K, the two upper bands and two down bands are almost doubly degenerate, respectively. This is different from the case of abca stacking in which the four bands of each spin channel are separated clearly. The phenomenon at VB-K happens in tree-layer and four-layer $\mathrm{MoS}_{2}$ may be explained due to the being of mirror symmetry in aba and abab stacking sequences. 

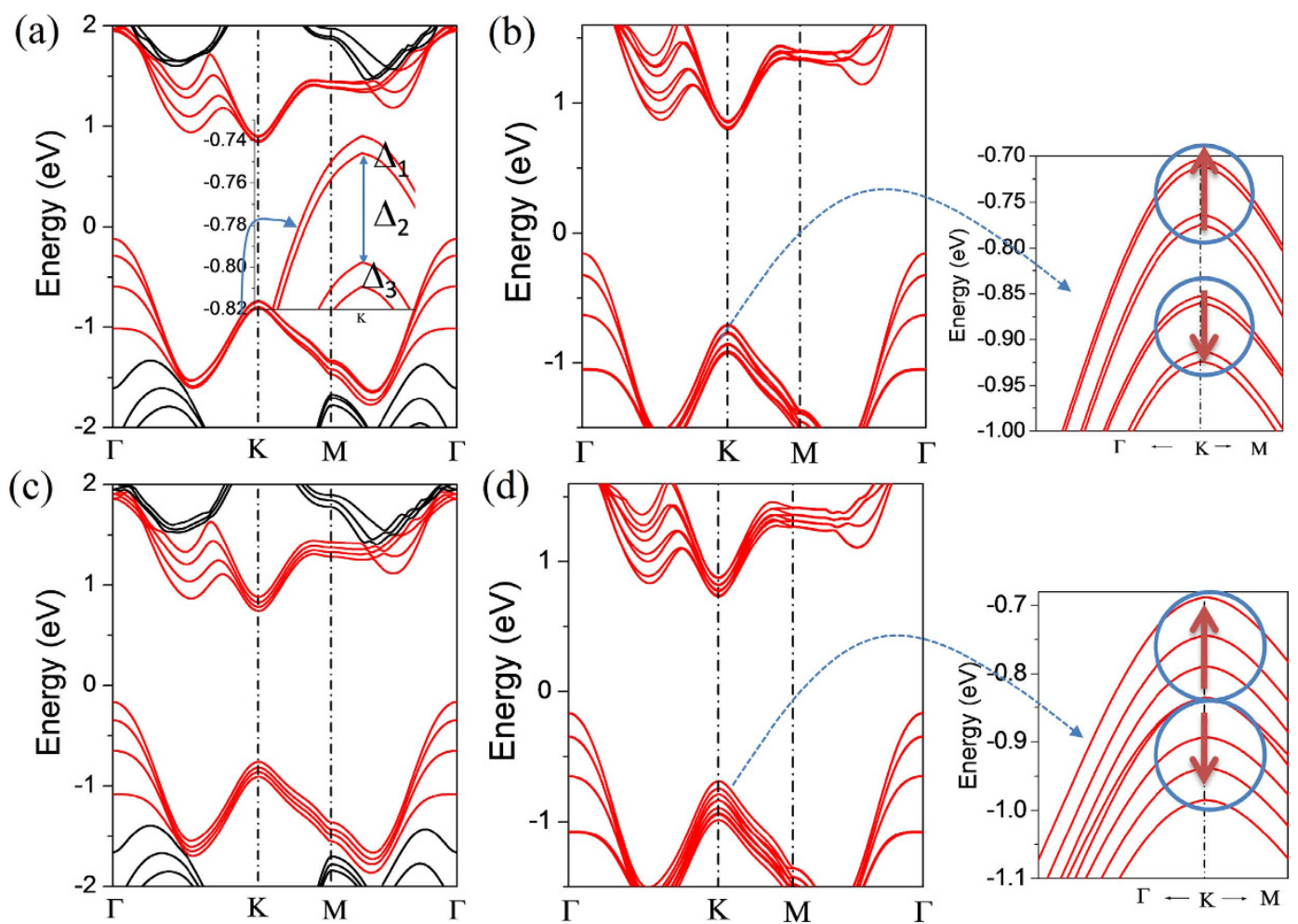

Figure 6. Band structures of 4-layer $\mathrm{MoS}_{2}$ with abab-stacking by calculated (a) without SOC and (b) with SOC, and with abca-stacking by calculated (c) without SOC and (d) with SOC. Note that in the inset of Fig. 6b and d, the band structure is plotted with two directions $\mathrm{K} \rightarrow \Gamma$ and $\mathrm{K} \rightarrow \mathrm{M}$ from the top of valance band near $\mathrm{K}$ point and the lengths for $\mathrm{K} \rightarrow \Gamma$ and $\mathrm{k} \rightarrow \mathrm{M}$ are the $1 / 10$ of total lengths in the two directions, respectively.

From the above analysis, the SOC effect is similar for different stacking orders. This may be because the spin-orbit interaction happens mainly in intra-layer and the SOC between layers can be ignored. It can be easily understood from geometry aspect, since the distance between Mo atoms in different layer are far away from each other, compared to that in the same layer. The difference of band splitting near band gap for different stacking orders can be attributed to the LC effect. In addition, it is found that the SOC doesn't impact the splitting from LC. The layers' interactions due to the different stacking orders just have obvious effects on the states near Fermi level. In Table 1, we list all the splitting values from LC for different layers and different stacking orders for the typical states VB- $\Gamma$, VB-K, CB- $\Lambda$ and CB-K. For double-layer, we can propose the simple model Hamiltonian to analyze the splitting by the formula,

$$
H(k)=\left(\begin{array}{cc}
E_{0}(k) & M_{1}(k) \\
M_{1}^{*}(k) & E_{0}(k)
\end{array}\right),
$$

where $\mathrm{M}_{1}(\mathrm{k})$ is the coupling parameter between the states from two layers in same $\mathrm{k}$ point with same energy level. From this model, we can find that the parameter $\mathrm{M}_{1}(\mathrm{k})$ is equal to a half of the splitting value of both level $(\Delta / 2)$ in Table 1. With this idea, we can analyze the coupling parameters of other stacking orders with different layers.

It is found that the nearest-neighbor coupling is not enough to understand the splitting of multi-layer and the coupling between layers which are far way with each other may need to be considered. With the model Hamiltonian in supplementary information, $M_{1}, M_{2}$ and $M_{3}$ represent the coupling parameters of nearest-neighbor, second near-neighbor and third near-neighbor layer-coupling, respectively. By these parameters to fit the splitting values from DFT calculation, we can obtain the values of these coupling parameters, as shown in Table 2. It can be found that the quantum coupling of the states at VB- $\Gamma$ and $C B-\Lambda$ is distinctly stronger than that at CB-K and VB-K. Generally, the value of nearest-neighbor coupling parameter is the largest among these parameters. By the comparison between the parameters of stacking order aba and abc for tree-layer $\mathrm{MoS}_{2}$, the nearest-neighbor coupling of abc stacking is stronger than that of aba stacking. At the same time, for four-layer $\mathrm{MoS}_{2}$, the nearest-neighbor coupling of abca stacking is stronger than that of abab stacking. Especially, this phenomenon is very obvious for the states at VB-K and CB-K. The stronger nearest-neighbor coupling for the stacking sequences abc and abca may give an implicature why the periodic abc stacking sequence (bulk $3 \mathrm{R}-\mathrm{MoS}_{2}$ ) is very popular than other stacking order, such as aba, abab, etc. The splitting of spin bands of each valley (K or/ and $\mathrm{K}^{\prime}$ ) in $3 \mathrm{R}$-type $\mathrm{MoS}_{2}$ is much different from that in $2 \mathrm{H}$-type $\mathrm{MoS}_{2}$ and can be detected by circular polarized photoluminescence as has been made in bulk 3R- $\mathrm{MoS}_{2}{ }^{35}$. For the different stacking orders of few-layer 3R-type $\mathrm{MoS}_{2}$, the splittings of valance bands in each spin channel of $\mathrm{K}(\mathrm{K})$ valley near Fermi level are evidently different 


\begin{tabular}{|l|c|c|c|c|c|c|c|c|c|c|c|c|}
\hline \multirow{2}{*}{$\begin{array}{l}\text { Stacking } \\
\text { way }\end{array}$} & \multicolumn{3}{|c|}{ VB- $\Gamma(\mathbf{e V})$} & \multicolumn{3}{c|}{ CB- $\boldsymbol{\Lambda}(\mathbf{e V})$} & \multicolumn{3}{c|}{ CB-K $\left(\mathbf{1 0}^{-\mathbf{2}} \mathbf{e V}\right)$} & \multicolumn{3}{c|}{$\mathbf{V B}_{\mathbf{K}}\left(\mathbf{1 0} \mathbf{1 0}^{-\mathbf{2}} \mathbf{e V}\right)$} \\
\cline { 2 - 14 } & $\mathbf{M}_{\mathbf{1}}$ & $\mathbf{M}_{\mathbf{2}}$ & $\mathbf{M}_{\mathbf{3}}$ & $\mathbf{M}_{\mathbf{1}}$ & $\mathbf{M}_{\mathbf{2}}$ & $\mathbf{M}_{\mathbf{3}}$ & $\mathbf{M}_{\mathbf{1}}$ & $\mathbf{M}_{\mathbf{2}}$ & $\mathbf{M}_{\mathbf{3}}$ & $\mathbf{M}_{\mathbf{1}}$ & $\mathbf{M}_{\mathbf{2}}$ & $\mathbf{M}_{\mathbf{3}}$ \\
\hline 2L-ab & 0.3194 & - & - & 0.1529 & - & - & 2.71 & - & - & 3.05 & - & - \\
\hline 3L-aba & 0.2835 & 0.0850 & - & 0.1432 & 0.0440 & - & 1.89 & 1.81 & - & 2.34 & 1.75 & - \\
\hline 3L-abc & 0.2515 & 0.0720 & - & 0.1485 & 0.0110 & - & 3.19 & -0.55 & - & 3.46 & -0.40 & - \\
\hline 4L-abab & 0.2725 & 0.0690 & 0.0230 & 0.1442 & 0.0165 & -0.0150 & 1.80 & 0.62 & -1.70 & 2.20 & 0.69 & -2.02 \\
\hline 4L-abca & 0.2770 & 0.0690 & 0.0295 & 0.1497 & 0.0095 & 0.0030 & 4.23 & -0.40 & 0.58 & 4.44 & -0.275 & 0.715 \\
\hline
\end{tabular}

Table 2. The values of coupling parameters from the model Hamiltonian about layers' coupling which are obtained by fitting the splitting values from Table 1 for $2 \mathrm{~L}$-ab, 3L-aba, 3L-abc, $4 \mathrm{~L}$-abab and $4 \mathrm{~L}$-abca with the different layers and stacking orders. Note that $\mathrm{M}_{1}, \mathrm{M}_{2}$ and $\mathrm{M}_{3}$ represent the nearest-neighbor, second nearneighbor and third near-neighbor layer-coupling parameters, respectively.

and expected to observe in high resolution photoluminescence and detect with high resolution angle-resolved photoemission spectroscopy.

\section{Conclusions and Outlook}

With first-principle method, we studied the effect of stacking orders on the electronic structures of 3R-type multi-layer $\mathrm{MoS}_{2}$ with spin-orbit interaction and layer's coupling. By simulating the gliding of single-layer $\mathrm{MoS}_{2}$ on the surface of the down layer of $\mathrm{MoS}_{2}$ and analyzing the energy surface, there are two energy minima with an energy barrier of about $18 \mathrm{meV}$ which correspond to two different configurations, like that happening in the low density phase of $\mathrm{BN}$. This makes it possible that there is a lot of stacking order sequences that makes rich polytypism of $\mathrm{MoS}_{2}$ and also gives rise to an issue why bulk $3 \mathrm{R}-\mathrm{MoS}_{2}$ is popular than other polytypes, besides $2 \mathrm{H}-\mathrm{MoS}_{2}$.

It is found that the band splitting of $\mathrm{CB}-\Gamma$ is just controlled by the LC, regardless the stacking orders and layers' number. The band splittings of CB- $\Lambda$ and CB-K are decided mainly by the LC with the perturbation of SOC. The band splitting of VB-K is mainly controlled by the SOC, and this makes that the spin-up and spin-down channels are separated clearly which will result in the clear valley dependent spin polarization in multi-layer 3R-type $\mathrm{MoS}_{2}$. With model Hamiltonian, the LC effect on band splitting is analyzed in detail. It is found that the stacking sequences, such as abc and abca, have the stronger nearest-neighbor coupling. This may imply the popular of the periodic abc stacking sequence in natural growth of $\mathrm{MoS}_{2}$, besides $2 \mathrm{H}-\mathrm{MoS}_{2}$.

Few-layer 3R-type $\mathrm{MoS}_{2}$, which is different from 2H-type, is without inversion symmetry and is a kind of promising material for valley/spin physics. In the $3 \mathrm{R}$-type $\mathrm{MoS}_{2}$, there is no rotation along $\mathrm{z}$ axis between layers and the separation between spin-up and spin-down channels in $\mathrm{K}$ (or/and $\mathrm{K}$ ') valley is obvious and isn't sensitive to the stacking orders. This promises the enhanced valley polarization is easily observed in the circular polarized photoluminescence. It is expected this work about 3R-type $\mathrm{MoS}_{2}$ can push forward the research the valley/spin physics on 2D materials.

\section{Methods}

The present calculations are performed within density functional theory using accurate frozen-core full-potential projector augmented-wave (PAW) pseudopotentials, as implemented in the VASP code ${ }^{40-42}$. We did calculations with the generalized gradient approximation (GGA) of Perdew, Burke and Ernzerhof (PBE) and with added van der Waals corrections ${ }^{43}$. The $k$-space integrals and the plane-wave basis sets are chosen to ensure that the total energy is converged at $1 \mathrm{meV} /$ atom level. A kinetic energy cutoff of $500 \mathrm{eV}$ for the plane wave expansion is found to be sufficient. The Brillouin zones are sampled with dense $\Gamma$-centered $18 \times 18 \times 1$ grids for supercell about the multi-layer model of $\mathrm{MoS}_{2}$. The effect of dispersion interaction is included by the empirical correction scheme of Grimme $(\mathrm{DFT}+\mathrm{D} / \mathrm{PBE})^{44}$. This approach has been successful in describing layered structures ${ }^{39,45}$.

The calculated lattice constants $a$ and $c$ of bulk $\mathrm{MoS}_{2}$ are $3.188 \AA$ and $12.402 \AA$, similar to the experimental values (3.160 $\AA$ and $12.295 \AA$ ). The calculated band gap of single-layer $\mathrm{MoS}_{2}$ without the consideration of spin-orbit interaction is $1.66 \mathrm{eV}$ and less than the experimental report of about $1.8 \mathrm{eV}^{1,46}$. Obviously, the band gap from PBE is underestimated as in common in usual density functional calculations. Though the band gap is underestimated by GGA/PBE, the band structure near Fermi level doesn't have obvious difference from that from other many body method. The analysis of the effect of spin-orbital coupling on the electronic states near band gap is enough with GGA/PBE ${ }^{47-52}$. The multilayer $\mathrm{MoS}_{2}$ sheets are simulated with the supercells with vacuum spaces of $15 \AA$ along $\mathrm{z}$ direction in order to avoid the spurious vertical coupling effect. It is noticed that the symmetry doesn't allow a net polarization or electric dipole along $\mathrm{z}$ in all the cases although the layered stacks haven't inversion. This is because of the mirror in the center of the central $\mathrm{MoS}_{2}$ sheet.

\section{References}

1. Mak, K. F., Lee, C., Hone, J., Shan, J. \& Heinz, T. F. Atomically thin $\mathrm{MoS}_{2}$ : a new direct-gap semiconductor. Phys. Rev. Lett. 105, 136805 (2010).

2. Wang, Q. H., Kalantar-Zadeh, K., Kis, A., Coleman, J. N. \& Strano, M. S. Electronics and optoelectronics of two-dimensional transition metal dichalcogenides. Nat. Nanotech. 7, 699-712 (2012).

3. Radisavljevic, B., Radenovic, A., Brivio, J., Giacometti, V. \& Kis, A. Single-layer $\mathrm{MoS}_{2}$ transistors. Nat Nanotech. 6, 147-150 (2011).

4. Lee, C. et al. Anomalous lattice vibrations of single- and few-layer $\mathrm{MoS}_{2}$. ACS Nano 4, 2695-2700 (2010).

5. Zhu, Z. Y., Cheng, Y. C. \& Schwingenschlögl, U. Giant spin-orbit-induced spin splitting in two-dimensional transition-metal dichalcogenide semiconductors. Phys. Rev. B 84, 153402 (2011).

6. Zeng, H., Dai, J., Yao, W., Xiao, D. \& Cui, X. Valley polarization in $\mathrm{MoS}_{2}$ monolayers by optical pumping. Nat. Nanotech. 7, 490-493 (2012). 
7. Cao, T. et al. Valley-selective circular dichroism of monolayer molybdenum disulphide. Nat Commun 3, 887 (2012).

8. Xiao, D., Liu, G.-B., Feng, W., Xu, X. \& Yao, W. Coupled spin and valley physics in monolayers of $\mathrm{MoS}_{2}$ and other group-VI dichalcogenides. Phys. Rev. Lett. 108, 196802 (2012).

9. Mak, K. F., McGill, K. L., Park, J. \& McEuen, P. L. The valley Hall effect in $\mathrm{MoS}_{2}$ transistors. Science 344, 1489-1492 (2014).

10. Roldán, R., Cappelluti, E. \& Guinea, F. Interactions and superconductivity in heavily doped MoS $_{2}$. Phys. Rev. B 88, 054515 (2013).

11. Geim, A. K. \& Grigorieva, I. V. Van der Waals heterostructures. Nature 499, 419-425 (2013).

12. Sun, L. et al. Spin-orbit splitting in single-layer $\mathrm{MoS}_{2}$ revealed by triply resonant Raman scattering. Physical Review Letters 111, 126801 (2013).

13. Alidoust, N. et al. Observation of monolayer valence band spin-orbit effect and induced quantum well states in $\operatorname{MoX}_{2}$. Nat Commun 5, 1312 (2014).

14. Mak, K. F. et al. Tightly bound trions in monolayer $\mathrm{MoS}_{2}$. Nat Mater 12, 207-211 (2013).

15. Ross, J. S. et al. Electrical control of neutral and charged excitons in a monolayer semiconductor. Nat Commun 4, 1474 (2013).

16. Klots, A. R. et al. Probing excitonic states in suspended two-dimensional semiconductors by photocurrent spectroscopy. Scientific Reports 4, 6608 (2014).

17. Splendiani, A. et al. Emerging photoluminescence in monolayer $\mathrm{MoS}_{2}$. Nano Lett. 10, 1271-1275 (2010).

18. Zhang, Y. et al. Direct observation of the transition from indirect to direct bandgap in atomically thin epitaxial $\mathrm{MoSe}_{2}$. Nat Nanotech 9, 111-115 (2014).

19. Yao, W., Xiao, D. \& Niu, Q. Valley-dependent optoelectronics from inversion symmetry breaking. Phys. Rev. B 77, 235406 (2008).

20. Nan, H. et al. Strong photoluminescence enhancement of $\mathrm{MoS}_{2}$ through defect engineering and oxygen bonding. ACS Nano 8, 5738-5745 (2014).

21. Mouri, S., Miyauchi, Y. \& Matsuda, K. Tunable photoluminescence of monolayer $\mathrm{MoS}_{2}$ via chemical doping. Nano Letters 5944-5948 (2013).

22. Tongay, S. et al. Defects activated photoluminescence in two-dimensional semiconductors: interplay between bound, charged, and free excitons. Scientific Reports 3, 2657 (2013).

23. Feng, J., Qian, X., Huang, C.-W. \& Li, J. Strain-engineered artificial atom as a broad-spectrum solar energy funnel. Nat. Photon. 6, 866-872 (2012)

24. Chang, C.-H., Fan, X. F., Lin, S.-H. \& Kuo, J.-L. Orbital analysis of electronic structure and phonon dispersion in $\mathrm{MoS}_{2}, \mathrm{MoSe}_{2}, \mathrm{WS}_{2}$, and $\mathrm{WSe}_{2}$ monolayers under strain. Phys. Rev. B 88, 195420 (2013).

25. Fan, X. F., Zheng, W. T., Kuo, J.-L. \& Singh, D. J. Structural stability of single-layer $\mathrm{MoS}_{2}$ under large strain. J. Phys. Condens. Matter 27, 105401 (2015).

26. Scalise, E., Houssa, M., Pourtois, G., Afanasev, V. \& Stesmans, A. Strain-induced semiconductor to metal transition in the twodimensional honeycomb structure of $\mathrm{MoS}_{2}$. Nano Research 5, 43-48 (2012).

27. Conley, H. J. et al. Bandgap engineering of strained monolayer and bilayer $\mathrm{MoS}_{2}$. Nano Lett. 13, 3626-3630 (2013).

28. Liu, K. et al. Evolution of interlayer coupling in twisted molybdenum disulfide bilayers. Nat Commun 5, 4966 (2014).

29. Luo, X. et al. Stacking sequence determines Raman intensities of observed interlayer shear modes in 2D layered materials - A general bond polarizability model. Scientific Reports 5, 14565 (2015).

30. Mattheiss, L. F. Band structures of transition-metal-dichalcogenide layer compounds. Phys. Rev. B 8, 3719-3740 (1973)

31. Fan, X., Zheng, W. T., Jiang, Q. \& Singh, D. J. Pressure evolution of the potential barriers for transformations of layered BN to dense structures. RSC Advances 5, 87550-87555 (2015).

32. Wilson, J. A. \& Yoffe, A. D. The transition metal dichalcogenides discussion and interpretation of the observed optical, electrical and structural properties. Advances in Physics 18, 193-335 (1969).

33. Huang, H. H. et al. Controlling phase transition for single-layer $\mathrm{MTe}_{2}(\mathrm{M}=\mathrm{Mo}$ and $\mathrm{W})$ : modulation of the potential barrier under strain. Phys. Chem. Chem. Phys. 18, 4086-4094 (2016).

34. Towle, L. C., Oberbeck, V., Brown, B. E. \& Stajdohar, R. E. Molybdenum diselenide: rhombohedral high pressure-high temperature polymorph. Science 154, 895-896 (1966).

35. Suzuki, R. et al. Valley-dependent spin polarization in bulk $\mathrm{MoS}_{2}$ with broken inversion symmetry. Nat Nano 9, 611-617 (2014).

36. Jiang, T. et al. Valley and band structure engineering of folded $\mathrm{MoS}_{2}$ bilayers. Nat Nano 9, 825-829 (2014).

37. Morpurgo, A. F. Ten years of Nature Physics: The ABC of 2D materials. Nat Phys 11, 625-626 (2015).

38. Yeh, P.-C. et al. Layer-dependent electronic structure of an atomically heavy two-dimensional dichalcogenide. Phys. Rev. B 91, 041407 (2015).

39. Fan, X. F., Chang, C. H., Zheng, W. T., Kuo, J.-L. \& Singh, D. J. The electronic properties of single-layer and multilayer $\mathrm{MoS}_{2}$ under high pressure. J. Phys. Chem. C 119, 10189-10196 (2015).

40. Hohenberg, P. \& Kohn, W. Inhomogeneous electron gas. Phys. Rev. 136, B864 (1964).

41. Kresse, G. \& Furthmüller, J. Efficient iterative schemes for ab initio total-energy calculations using a plane-wave basis set. Phys. Rev. $B$ 54, 11169-11186 (1996).

42. Kresse, G. \& Furthmüller, J. Efficiency of ab-initio total energy calculations for metals and semiconductors using a plane-wave basis set Computat. Mater. Sci. 6, 15-50 (1996).

43. Perdew, J. P., Burke, K. \& Ernzerhof, M. Generalized gradient approximation made simple. Phys. Rev. Lett. 77, 3865-3868 (1996).

44. Grimme, S. Semiempirical GGA-type density functional constructed with a long-range dispersion correction. J. Comput. Chem. 27, 1787 (2006).

45. Mercurio, G. et al. Structure and energetics of azobenzene on $\mathrm{Ag}(111)$ : benchmarking semiempirical dispersion correction approaches. Phys. Rev. Lett. 104, 036102 (2010).

46. Kuc, A., Zibouche, N. \& Heine, T. Influence of quantum confinement on the electronic structure of the transition metal sulfide $\mathrm{TS}_{2}$. Phys. Rev. B 83, 245213 (2011).

47. Hromadová, L., Martoňák, R. \& Tosatti, E. Structure change, layer sliding, and metallization in high-pressure MoS 2. Phys. Rev. B 87, 144105 (2013).

48. Latzke, D. W. et al. Electronic structure, spin-orbit coupling, and interlayer interaction in bulk $\mathrm{MoS}_{2}$ and $\mathrm{WS}_{2}$. Phys. Rev. B 91, 235202 (2015).

49. Kang, J., Li, J., Li, S.-S., Xia, J.-B. \& Wang, L.-W. Electronic structural Moire pattern effects on $\mathrm{MoS}_{2} / \mathrm{MoSe}_{2} 2 \mathrm{D}$ heterostructures. Nano Lett. 13, 5485-5490 (2013).

50. Eknapakul, T. et al. Electronic structure of a quasi-free standing $\mathrm{MoS}_{2}$ Monolayer. Nano Letters 14, 1312-1316 (2014).

51. Dou, X., Ding, K., Jiang, D., Fan, X. \& Sun, B. Probing spin-orbit coupling and interlayer coupling in atomically thin molybdenum disulfide using hydrostatic pressure. ACS Nano 10, 1619-1624 (2016).

52. Chang, T.-R., Lin, H., Jeng, H.-T. \& Bansil, A. Thickness dependence of spin polarization and electronic structure of ultra-thin films of $\mathrm{MoS}_{2}$ and related transition-metal dichalcogenides. Scientific Reports 4, 6270 (2014).

\section{Acknowledgements}

The authors would like to acknowledge the support by Nanyang Technological University under Grant Nos RG97/15 and RG101/14 and National Natural Science Foundation of China under Grant No. 11504123 for this research. 


\section{Author Contributions}

X.F. did first principles calculations. X.F., W.T.Z., J.L.K., D.J.S. and W.Z. contributed to the concept and analysis of results. X.F., C.Q.S. and W.Z. participated in drafting the manuscript.

\section{Additional Information}

Supplementary information accompanies this paper at http://www.nature.com/srep

Competing financial interests: The authors declare no competing financial interests.

How to cite this article: Fan, X. et al. Modulation of electronic properties from stacking orders and spin-orbit coupling for 3R-type $\mathrm{MoS}_{2}$. Sci. Rep. 6, 24140; doi: 10.1038/srep24140 (2016).

(c) (i) This work is licensed under a Creative Commons Attribution 4.0 International License. The images or other third party material in this article are included in the article's Creative Commons license, unless indicated otherwise in the credit line; if the material is not included under the Creative Commons license, users will need to obtain permission from the license holder to reproduce the material. To view a copy of this license, visit http://creativecommons.org/licenses/by/4.0/ 